\title{
Mathematical Modeling and Design Considerations for Designing Voice Coil Motor
}

\author{
Jae-heon Jeong ${ }^{1,} \mathrm{a}^{*}$, MyeongHyeon Kim ${ }^{1}$, Jaehyun Park ${ }^{1}$, Daegab Gweon ${ }^{1}$ \\ ${ }^{1}$ Nano-Opto-Mechatronics Lab., Department of Mechanical Engineering, KAIST, \\ 291 Daehak-ro, Yuseong-gu, Daejeon, 34141, South Korea \\ apkwogjs007@kaist.ac.kr
}

Keywords: Voice coil motor, design, mathematical modeling

\begin{abstract}
The voice coil motor is a device motivated by electric current. It is creating force by Lorentz force principle generated by interaction of the current and magnetic field from magnets. The voice coil motor has been used for various purpose such as speaker, actuator, and etc. Especially, there has been numerous types of voice coil motors for actuators. Among those, a common type of voice coil motor which has wounded coil in the center covered with Halbach magnet array with iron yoke is object system of interest to be mathematically modeled. In this paper, the voice coil motor of the type is to be mathematically designed with Lorentz force principle. Since the Lorentz force is generated from current flowing in coil and magnetic field from magnets, method for analysis of magnetic field and of coil is described. Lorentz force is proportional to current. Therefore, final design variable to be found is a motor constant which is ratio of force to current.
\end{abstract}

\section{Introduction}

Voice coil motor (VCM) is a device motivated by electric current creating Lorentz force generated by interaction of the current and magnetic field from magnets. The VCM has been used for various purpose such as speaker, actuator, and etc. Especially, the VCM plays important role as actuators in precision positioning system [1] or active vibration isolation [2]. The usage of the VCM is getting increased with time. In this paper, one of effective type of VCM is studied [3]. Firstly, geometrical concept of the VCM is described. Then, a motor constant of the VCM is mathematically modeled with Lorentz force equation. Derivation of the Lorentz force of the VCM is studied. Furthermore, since the Lorentz force includes mathematical integration of magnetic field intensity over area, methods for calculating integration of the magnetic field intensity is introduced. Finally, relation between coil-passing area and suppressing parasitic motion is described, which can be design consideration for deciding the coil-passing area.

\section{Geometrical concept of VCM}

Geometrical concept of the VCM which is object system in this paper is shown in Fig. 1. Magnet groups are located above a coil which is positioned in the center of the VCM and under the coil. The magnet groups have iron yoke on three magnets for increasing magnetic field toward to the coil. Arrow indices on the magnets are indicating magnetization direction of the magnets. It is noted that magnetization of the middle magnet is horizontal direction unlike the other magnets. This arrangement is called Halbach magnet array [4] which is invented for increasing magnet field intensity of intended direction while magnetic field of the opposite direction is weakened. The magnet array were set to improve the magnetic field of magnets toward the coil side. This magnet array allows almost $\mathrm{z}$ directional component of magnetic field being applied to the coil because of tendency of magnetic field distribution shown in Fig. 6. 


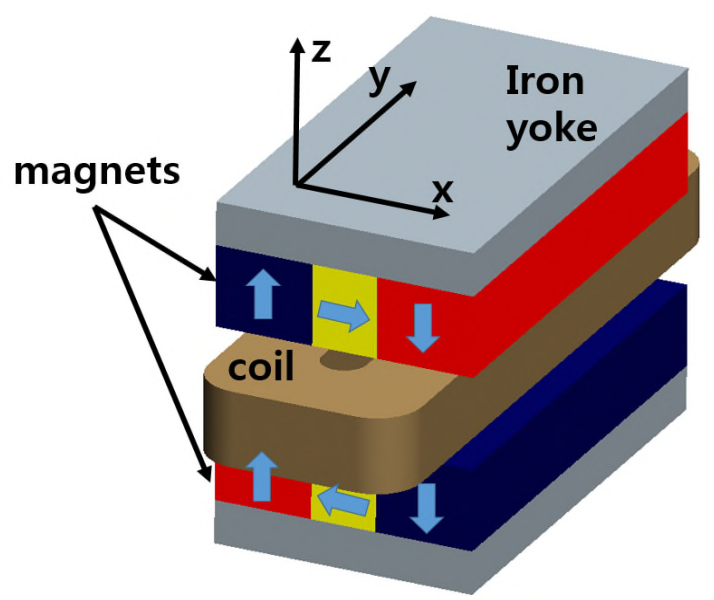

Fig. 1 Geometrical concept of the VCM.

Fig. 2 shows geometrical shape of coil. Middle part of the coil is stretched and tips of the coil are rounded. The rounded tips can make force analysis complicated because current is flowing through the rounded part which means coil is flowing in two dimensional plane. Furthermore, current flowing in the rounded part generates parasitic force which is not intended force. Therefore, it is best decision that the only stretched part is covered by magnets, not the rounded part because force is not generated if there is no magnetic field applied to the part of the coil.

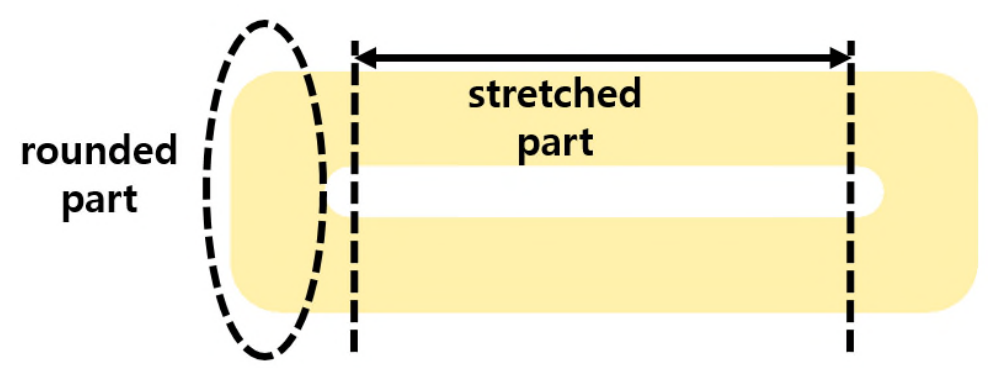

Fig. 2 Geometrical shape of coil.

\section{Mathematical Modeling of the Motor Constant of the VCM with Lorentz Force Equation}

In order to mathematically model the motor constant of the VCM, a governing force equation is necessary. For governing force equation of the VCM, Lorentz force equation is used below where $\vec{F}$ is Lorentz force vector, $\vec{J}$ is current density vector, $\vec{B}$ is magnetic field intensity vector, and $V$ is volume of the stretched part of the coil.

$$
\vec{F}=\int_{V} \vec{J} \times \vec{B} d V
$$

Since the only stretched part of the coil is to be integrated, $\vec{J}$ vector can be treated as constant along the y axis integral. Also, $\vec{B}$ is not changing along the y axis integral because the magnet is stretched along the same direction. Therefore, the volume integral can be divided in to two integrals: surface integral and line integral over length of the stretched part of the coil, $l$.

$$
\vec{F}=\int_{l} \int_{A} \vec{J} \times \vec{B} d A d l
$$

$\vec{J}$ vector can be substituted with $\vec{I}$ vector divided by cross-sectional area of the coil in $\mathrm{x}-\mathrm{z}$ plane, $A$ (defined coil-passing area in this paper), where $\vec{I}$ vector is current vector flowing in the area. Since the magnets are stretched along the y axis, y directional magnetic field generated by the magnets can be neglected. Therefore, the magnet field intensity vector can be decomposed with Cartesian coordinate. 


$$
\vec{F}=\frac{1}{A} \iint_{l} \int_{A} \vec{I} \times\left(B_{x} \vec{x}+B_{z} \vec{z}\right) d A d l
$$

Since the $\vec{I}$ vector is ether positive y direction or negative y direction, $\vec{I}$ is either $-|\vec{I}| \vec{y}$ or $|\vec{I}| \vec{y}$. With letting the current flows clockwise direction when the VCM is shown from positive $\mathrm{z}$ axis, $\vec{I}$ vector in left coil block and right coil block in Fig. 3 are $|\vec{I}| \vec{y}$ and $-|\vec{I}| \vec{y}$, respectively. Therefore, the equation can be rewritten as below.

$$
\vec{F}=\frac{l|\vec{I}|}{A} \int_{A}\left(-B_{x, l e f t} \vec{z}+B_{z, l e f t} \vec{x}\right) d A+\frac{l|\vec{I}|}{A} \int_{A}\left(B_{x, r i g h t} \vec{z}-B_{z, r i g h t} \vec{x}\right) d A
$$

In the $x-z$ plane, the magnet groups are placed by origin symmetry each other which origin is the center of the VCM. Therefore, if the coil is also place by origin symmetry like the magnet groups, the magnetic field intensity in the left coil block and the right coil block is also placed by origin symmetry, which simplifies the equation into:

$$
\vec{F}=2 \frac{l|\vec{I}|}{A} \int_{A}\left(-B_{x, l e f t} \vec{z}+B_{z, l e f t} \vec{x}\right) d A
$$

Since the $|\vec{I}|$ is total current flowing in the coil, the term can be expressed with the number of coil turn times current flowing in a single wire of the coil. The number of coil turn, $n$, is decided by geometrical dimensions of coil shown below

$$
n=\left(\frac{w_{c}}{d_{c}}-\frac{1}{2}\right)\left[\frac{2}{\sqrt{3}}\left(\frac{t_{c}}{d_{c}}-1\right)+1\right]
$$

where $i$ is current flowing in a single wire of the coil, $w_{c}$ is width of the coil, $d_{c}$ is coil diameter of a single wire of the coil, and $t_{c}$ is thickness of the coil. Therefore, final equation of Lorentz force of the VCM is written below.

$$
\vec{F}=2\left(\frac{w_{c}}{d_{c}}-\frac{1}{2}\right)\left[\frac{2}{\sqrt{3}}\left(\frac{t_{c}}{d_{c}}-1\right)+1\right] \frac{l i}{A} \int_{A}\left(-B_{x, l e f t} \vec{z}+B_{z, l e f t} \vec{x}\right) d A
$$

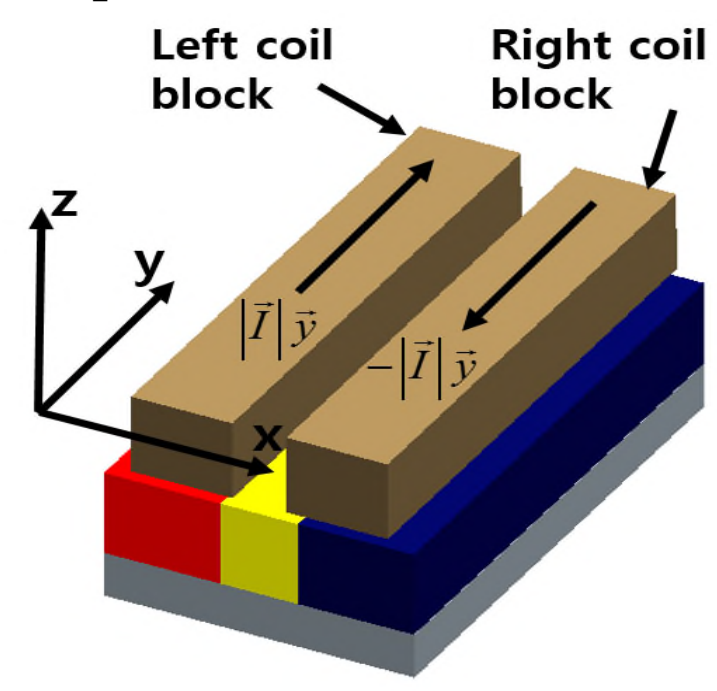

Fig. 3. Blocks of the coil which is to be integrated for calculating Lorentz force.

It is note that the Lorentz force in above equation is proportional to current flowing in a single wire of the coil. This can mean that the force can be infinitely maximized with infinitely high current. Therefore, a motor constant which is ratio of the force generated by the VCM to the current flowing in a single wire of the coil, instead of foce intensity itself, is more reasonable variable to represent effectness of design of the VCM. 
The parasitic force generated by integration value of $\mathrm{x}$ component of magnetic field is not variable interest. Furthermore, integration value can be switched to be average value over the coil-passing area because there is $\mathrm{A}$ in denominator in the equation. The final motor consant without parasitic force component is shown below.

$$
\frac{\vec{F}_{x}}{i}=2 l\left(\frac{w_{c}}{d_{c}}-\frac{1}{2}\right)\left[\frac{2}{\sqrt{3}}\left(\frac{t_{c}}{d_{c}}-1\right)+1\right] \frac{\int_{A} B_{z, l e f t} d A}{A}
$$

The final equation is expressed with geometrical value of coil and average value of magnetic field intensity over the coil-passing area.

\section{Magnetic Field Modeling of the Coil-Passing Area}

To find magnetic field of the coil-passing area, surface current modeling method [5] is used. The surface current modeling method is assuming that current is flowing on surfaces of the magnet with counter clockwise direction when the magnet is seen from magnetized direction. Since there is a mathematical equation of magnetic field generated from current, called Biot-Savart law, final magnetic field intensity on a certain point of interest can be calculated by integrating magnetic field generated from infinitesimally small current flowing on the surface. The final equation of the magnetic field intensity is written below when the coordinate and variables are shown in Figure 4.

$$
\begin{aligned}
& B_{x}=\int_{0}^{h} d B_{x}=-\left.\frac{K}{2}[G(a-x, y, z)+G(a-x, b-y, z)-G(x, y, z)-G(x, b-y, z)]\right|_{0} ^{h} \\
& B_{y}=\int_{0}^{h} d B_{y}=-\left.\frac{K}{2}[G(b-y, x, z)+G(b-y, a-x, z)-G(y, x, z)-G(y, a-x, z)]\right|_{0} ^{h} \\
& B_{z}=\int_{0}^{h} d B_{z}=-\left.K\left[\begin{array}{l}
H(y, a-x, z)+H(b-y, a-x, z)+H(x, b-y, z)+H(a-x, b-y, z) \\
+H(b-y, x, z)+H(y, x, z)+H(a-x, y, z)+H(x, y, z)
\end{array}\right]\right|_{0} ^{h} \\
& G(g 1, g 2, g 3)=\ln \left(\frac{\sqrt{g_{1}^{2}+g_{2}^{2}+\left(g_{3}^{2}-z_{0}\right)}-g_{2}}{g_{1}^{2}+g_{2}^{2}+\left(g_{3}^{2}-z_{0}\right)+g_{2}}\right) \\
& H\left(h_{1}, h_{2}, h_{3}\right)= \begin{cases}\arctan \left[\frac{h_{1}}{h_{2}} \frac{h_{3}-z_{0}}{\sqrt{h_{1}^{2}+h_{2}^{2}+\left(h_{3}-z_{0}\right)^{2}}}\right], & \text { if } y \neq 0 \\
0 & \text { if } y=0\end{cases}
\end{aligned}
$$

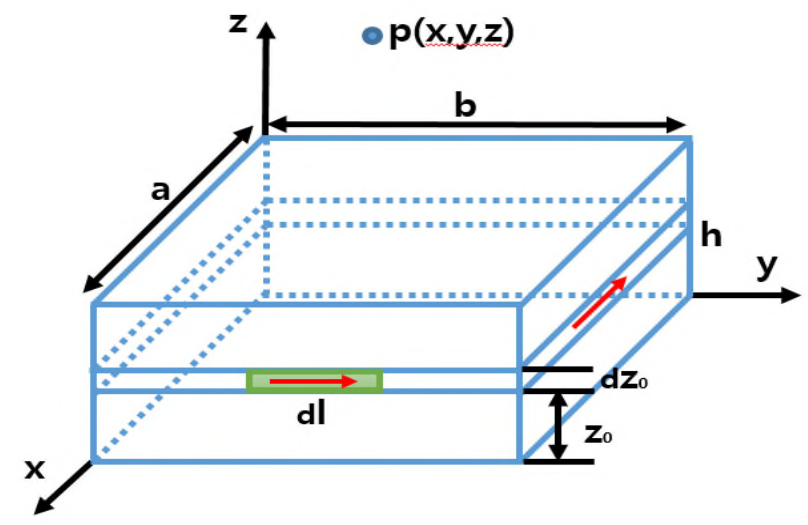

Fig. 4. Coordinate and variables for surface current modeling method. 
Before finding magnetic field of coil-passing area, analysis method for interpretation of iron yoke is needed. For the analysis, method of image is used. The method of image explains that when the iron yoke with infinite permeability is attached to magnets with magnetized direction, which is same case of blue and red magnets in Fig. 1, the magnets can be equal to magnets with doubled length with magnetized direction shown in Fig. 5.
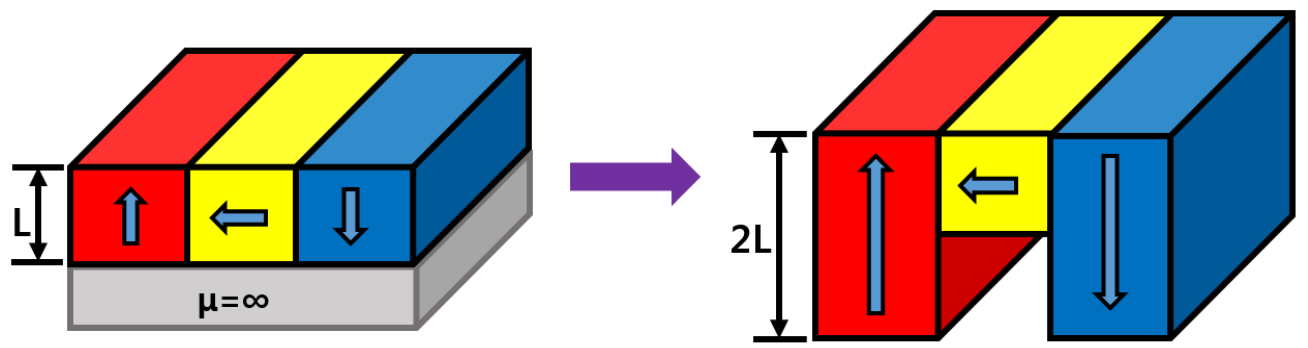

Fig. 5 Interpretation of iron yoke with method of image.

Since there are six magnets in the VCM, the magnetic field of the coil-passing area is expressed as sum of each magnetic field intensity of the six magnets. For simplifying the summing process, coordinate transformation is used. The coordinate transformation is, firstly, translation towards to the origin of the absolute coordinate. Secondly, rotation is carried out for matching the direction of the magnetization of the magnets. Finally, translation to the position of the magnets showing in Fig. 5 is performed.

\section{Considerable Factors for Deciding Coil-Passing Area}

In Eq. 7, it is noted that the force generated by the VCM is composed of $\mathrm{x}$ directional component and $\mathrm{z}$ directional component. Since the only $\mathrm{x}$ directional component of the force from the VCM is intended, the $\mathrm{z}$ directional component of the force can be seen as parasitic force which is unwanted. The equation of $\mathrm{z}$ directional component of the force is shown below.

$$
\vec{F}_{z}=2 \frac{l|\vec{I}|}{A} \int_{A}\left(-B_{x, l e f t} \vec{z}\right) d A
$$

In the equation, it is noted that the $\mathrm{x}$ directional component of magnetic field is cause of the unwanted force. Therefore, designing process should be minimizing integrated value of $\mathrm{x}$ directional component of magnetic field over coil-passing area. Since the integrated value should be minimized, either the $\mathrm{x}$ directional component of the magnetic field should be absolutely minimized or the integrated value should be minimized. This can be possible by deciding coil-passing area properly to make the integrated value minimized. Fig. 6 shows computationally simulated magnetic field intensity on the $\mathrm{x}-\mathrm{z}$ plane of the VCM in Fig. 1 with randomly chosen geometrical dimensions of magnets and iron yoke. In the center of the area where is supposed to be coil in, which is marked with red rectangular in Fig. 6, most of the component of the magnet field intensity is $\mathrm{z}$ directional component. As the coil is thickened with $\mathrm{z}$ direction, the coil-passing area is including more $\mathrm{z}$ directional component of the magnet field intensity, which enhance force of the VCM, while it is also including $\mathrm{x}$ directional component of the magnetic field. Similarly, as the coils is thickened with $\mathrm{x}$ directional component, the coil-passing area is also including more $\mathrm{x}$ directional component of magnetic field intensity. However, in this case, it is including much more $\mathrm{x}$ directional component of the magnetic field intensity than thickening with $\mathrm{z}$ direction does. Since the integrated value of $\mathrm{z}$ component of magnetic field intensity is getting bigger as the coil-passing area is getting larger, expanding coil-passing area as much as possible seems to be best way for improving Lorentz force. However, expanding coil-passing area is not a good idea for suppression of parasitic force. Therefore, optimization process is necessary for improving high Lorentz force with tolerable parasitic force of the VCM. 


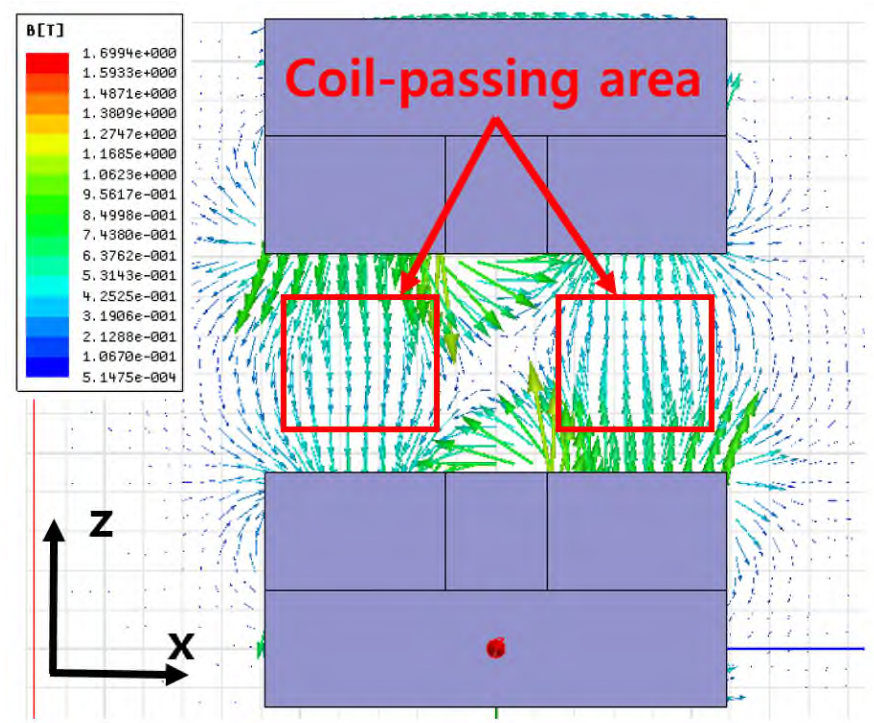

Fig. 6 Computationally simulated magnetic field intensity on the x-z plae of a VCM.

\section{Conclusion}

In this paper, method of mathematical modeling of mostly used type of VCM is studied. The modeling is based on mathematical expression of Lorentz force. Since the Lorentz force is generated from the current flowing in coil and magnetic field intensity from magnets with yoke, magnetic field from magnets with yoke and effect of geometrical dimension of coil were analyzed. For finding magnetic field, surface current model which basic idea is that magnetic field is generated from virtual current flowing on the surface of the magnets. To figure out effect of iron yoke, method of image is used. Since the maximum allowable current is depending on heat transferring and cooling problem of coil, motor constant which is ratio of force to current is chosen to final design variable. Finally, the motor equation can be expressed as geometrical dimensions of coil and magnets. The methodology proposed in this paper is expected to be used for designing this type of voice coil motor for future research.

\section{Acknowledgement}

This work was supported by the Technology Innovation Program (10040416, Technical development for ultra-precision position control considering micro vibration control) funded By the Ministry of Trade, industry \& Energy (MI, Korea)

\section{References}

[1] K. H. Kim, et al. Design of decoupled dual servo stage with voice coil motor and linear motor for XY long stroke ultra-precision scanning system, SPIE 6040, ICMIT 2005: Mechatronics, MEMS, and Smart Materials, 2005.

[2] R. Banik, D. G. Gweon. Design and optimization of voice coil motor for application in active vibration isolation, Sens. Actuat. A: Phys. 137(2) (2007) 236-243.

[3] D. H. Ahn. Dual servo planar motion stage using magnetic levitation technology. Ph.D. Dissertation. 2014.

[4] K. Halbach. Design of permanent multipole magnets with oriented rare earth cobalt material. Nuclear Instr. Meth. 169(1) (1980) 1-10.

[5] X. F. Gou et al. Analytic expression of magnetic field distribution of rectangular permanent magnets. Appl. Math. Mech. 25(3) (2004) 297-306. 